\title{
A high fat diet modulates expression of nutrient sensing receptors differentially throughout the gastrointestinal tract in mice
}

\author{
E. L. Symonds ${ }^{1}$, A. J. Page ${ }^{1,2}$, H. Li ${ }^{1,2}$ and L. A. Blackshaw ${ }^{1,2}$ \\ ${ }^{1}$ Nerve-Gut Research Laboratory, Hanson Institute, Royal Adelaide Hospital, Adelaide, South Australia, Australia and \\ ${ }^{2}$ Discipline of Medicine, University of Adelaide, Adelaide, South Australia, Australia
}

Recent work has shown that the G-protein coupled receptors involved in lingual taste detection of carbohydrates, fats and proteins are also found in the gut epithelium ${ }^{(1)}$, with our work showing them to be located throughout the entire length of the gastrointestinal tract ${ }^{(2)}$. This suggests an important role of the intestine in nutrient detection and appetite regulation, however little is known of the control of nutrient sensing receptor expression in response to a high fat diet. Previous studies have shown that a high fat diet can alter subsequent gastrointestinal responses to the presence of $\mathrm{fat}^{(3)}$. The mechanisms for this have not been elucidated but may involve alterations to the nutrient sensing receptors. This study therefore aimed to assess changes in receptor expression in the proximal and distal intestine after a high fat diet in mice.

Mucosal scrapings from the proximal and distal small intestine and the colon were collected from female C57BL/6 mice fed either a standard laboratory diet or a high fat diet (HDF, $60 \%$ energy from fat) for 12 weeks ( $n=7 /$ group). Quantitative RT-PCR was used to determine the relative expression of G-protein coupled receptors thought to play a role in sensing of carbohydrates (T1R2/T1R3), amino acids (T1R1/T1R3, Grm4, CaSR, GPR93) and fatty acids (GPR40, GPR41, GPR43, GPR84, GPR120). Results were expressed relative to Gapdh expression and compared between groups with the Wilcoxon signed-rank test.

Mice who had consumed the HFD had a significantly lower level of gene expression of carbohydrate (reduced to $0.1 \%$ of control levels) and amino acid (reduced to $29-44 \%$ of control levels) sensing receptors in the proximal small intestine $(P<0.05)$ compared to mice on a standard diet. Jejunal expression of short (GPR41, GPR43) and medium chain (GPR84) fatty acid sensing receptors were unchanged by the high fat diet, however expression of GPR40 and GPR120 (activated by long chain fatty acids) were down-regulated (reduced to $43 \%$ and $26 \%$ of control levels respectively, $P<0.05$ ). In the ileum there was a significant increase in short and medium chain fatty acid sensor expression (300 and $335 \%$ of control levels respectively, $P<0.05$ ), whilst there were no significant differences in receptor expression in the colon.

This study has revealed that proximal intestinal expression of amino acid, carbohydrate and long chain fatty acid sensing receptors is down-regulated by a high fat diet, whereas expression of the short and medium chain fatty acid sensing receptors is upregulated in the ileum. These changes could be induced by prolonged exposure to high fat and may contribute to altered gastrointestinal responses to fat that have been previous reported. This could adversely affect appetite and food intake regulation, and further contribute to the development of obesity.

This work was supported by AstraZeneca.

1. Höfer D, Püschel B \& Drenckhahn D (1996) Proc Natl Acad Sci USA 93, 6631-6634.

2. Symonds EL, Young RL, Page AJ et al. (2011) Gastroenterol 140, S334.

3. Boyd KA, O'Donovan DG, Doran S et al. (2003) Am J Physiol Gastrointest Liver Physiol. 284, G188-196. 